\title{
Preventive Effects of Fluoride Gel Application on Dental Caries in the Permanent Tooth: A Systematic Review of Randomized Controlled Trials
}

\author{
Kyung-Yi Do ${ }^{1, \ddagger}$ and Eun-Sun Lee $e^{2, \dagger, \ddagger}$ \\ 'Department of Public Health, Graduate School, The Catholic University of Korea, Seoul 06591, \\ ${ }^{2}$ Department of Dental Hygiene, Kyungbok University, Pocheon 11138, Korea
}

\begin{abstract}
Background: The purpose of this systematic review was to investigate the effects of topical fluoride gel application on dental caries prevention in the permanent teeth of children and adolescents.

Methods: We searched the EMBASE, PubMed, and Cochrane Library databases for randomized controlled trials (RCTs) assessing the effects of self-applied or professionally applied topical fluoride gels in patients <18 years of age; the search was completed on April 1, 2018. All included trials involved an experimental group (fluoride gel application) and a control group (placebo or no treatment). The outcome measures were the $D(M) F S$ and D(M)FT indices, which were compared between the two groups. Review Manager software was used for quantitative synthesis of the final selected articles, and a forest plot was generated via a meta-analysis conducted using a random effects model.

Results: The results showed that the $\mathrm{D}(\mathrm{M}) \mathrm{FS}$ and $\mathrm{D}(\mathrm{M}) \mathrm{FT}$ indices were lower in the fluoride application group than in the control group, thus indicating that fluoride gel application was effective in dental caries prevention. We also performed a subgroup analysis to determine whether the effects of fluoride application differed if patients received oral prophylaxis (self or professional) before fluoride gel application. Therefore, the two groups showed slightly larger differences when studies without oral prophylaxis before fluoride gel application were considered; however, the difference was not statistically significant.

Conclusion: These findings should be utilized to raise awareness about the caries-prevention effects of topical fluoride application among patients and guardians. Further RCTs should evaluate the effects of fluoride application with or without preceding oral prophylaxis, and appropriate fluoride application guidelines should be developed to maximize the effects of fluoride application in clinical practice.
\end{abstract}

Key Words: Dental caries, Fluorides, Randomized controlled trial, Systematic review

\section{Introduction}

Dental caries has been showing a gradually decreasing trend over the past few decades; however, it still affects $9 \%$ or approximately 610 million children worldwide ${ }^{1,2)}$. It is a chronic oral disease with a high prevalence among not only adults but also school-going children and adolescents. If timely treatment is not administered, caries can destroy the tooth crown and cause severe pain, thus having a negative impact on mastication and the quality of life in children and adolescents ${ }^{1)}$.

Dental caries is caused by tooth demineralization, whereby organic acids produced by oral resident microbiota and acids formed by plaque-induced decomposition of sugar in sweet foods destroy the tooth enamel. Daily tooth-brushing may slow the progression of caries, although the disease will continue to progress in most children $^{3,4)}$. Therefore, caries prevention is important. In addition to regular tooth-brushing after meals, regular check-ups and preventive treatment using sealants and 
topical fluorides are also recommended.

Topical fluoride application is widely recommended as part of dental caries prevention programs for school-going children, and it has been reported to be effective in the prevention of initial caries as well as the arrest of moderate to severe caries ${ }^{5}$. The home use of fluorides began with recommendations to use fluoride-containing toothpastes in the mid-1970s, while various caries-prevention strategies such as topical fluoride varnish application, liquid fluoride gargles, and xylitol intake have been used as additional measures. In particular, fluoride gel has been widely used in clinical settings because of the excellent costeffectiveness ${ }^{5,6)}$. Some existing studies have reported that the rate of caries prevention by fluoride application varies between $20 \%$ and $30 \%^{7-10)}$, whereas other randomized clinical trials have reported no differences between fluoride application and control groups ${ }^{11)}$. Even now, topical fluoride application for the prevention of dental caries shows different reliability and effects depending on the study method (cross-section, interventional, and randomized controlled trial [RCT] studies) and the quality control measures in the study. Moreover, awareness about the effects of topical fluoride application is also lacking. Marinho et al. ${ }^{1)}$ published a systematic review in 2015; however, the most recent study included in the review was conducted in 2005. Accordingly, we believe that there is a need for a systematic review including the most recent studies in order to derive the latest evidence. In addition, studies analyzing the effects of topical fluoride application after professional (oral prophylaxis) or self-cleaning (tooth-brushing) of teeth are rare.

Therefore, in order to present the latest and best evidence, we designed the present systematic review such that it included only RCTs assessing the caries-prevention effects of topical fluoride gels that are most widely used in the clinical setting. The main objective of this review was to address the following question: "Is topical fluoride gel application on the permanent teeth of children and adolescents effective in preventing dental caries?" The secondary objective was to address the following question: "Do the effects of topical fluoride gel application differ if it is implemented after professional (oral prophylaxis) or self-cleaning (tooth-brushing) of teeth?" Thus, the focus of this review was to verify the caries-prevention effects of topical fluoride gel application on the permanent teeth of children and adolescents.

\section{Materials and Methods}

\section{Subjects}

The present systematic review included preceding studies involving adolescents and children aged $<18$ years. To focus on the main questions (patient, intervention, comparison, outcome; PICO) of the study, only RCTs assessing the effects of self-applied or professionally applied topical fluoride gels on the decayed, missing, and filled permanent teeth (D(M)FT) and decayed, missing, and filled permanent tooth surface $(\mathrm{D}(\mathrm{M}) \mathrm{FS})$ indices were included. Studies were selected regardless of the severity of dental caries at the start of the study, the level of dental treatment, and ethnicity. Only those studies including subjects free of any systemic diseases were selected.

\section{Search strategy}

To select eligible RCTs, we used keywords from the main questions in PICO to search three core databases: PubMed, EMBASE, and the Cochrane Library. Moreover, to increase the sensitivity and specificity, we performed repeated searches according to a comprehensive search strategy. Search terms were selected from the MeSH (Medical Subject Headings) browser, and even Entree terminology, a sub-search terms of MeSH terminology was used. Moreover, keywords associated with the core subject of this review, which were identified through a search of the existing literature, were also added and combined with search operators (or/and). The search was completed on April 1, 2018. All databases were comprehensively searched for the earliest to the latest studies, regardless of the publication year.

\section{Inclusion criteria}

The inclusion criteria for studies were as follows: RCTs; assessment of the caries-prevention effects of self-applied or professionally applied topical fluoride gels on permanent teeth; inclusion of children and adolescents as subjects $(<18$ years); methods section specifically 
mentioning about random allocation of subjects to experimental and control groups; examination surveys independently performed by the investigator, with measurements derived under blinded conditions; detailed descriptions of the treatment method as well as the control or placebo intervention; a minimum follow-up period of 1 year; and analysis of the $\mathrm{D}(\mathrm{M}) \mathrm{FT}$ and $\mathrm{D}(\mathrm{M}) \mathrm{FS}$ indices as outcome measures. The type or concentration of the fluoride gel, the duration of topical application, and the frequency of topical application (at least once a year) were not criteria for inclusion.

\section{Exclusion criteria}

The exclusion criteria for this systematic review were as follows: case reports, editorials, and commentaries; literature not based on RCTs; no mention of random allocation of experimental and control groups; inclusion of subjects who were wearing orthodontic bands; a follow-up period of less than 1 year; lack of assessment of outcome indices; use of other forms of fluoride, such as varnishes and mouth rinses; and publication in languages other than English. Moreover, studies where subjects were receiving other types of treatment (chlorhexidine, sealant, xylitol, and other oral hygiene intervention) together were excluded.

\section{Selection of studies}

Two researchers selected the studies for this review. The identified studies were saved in EndNote program (ver. X7.5), followed by automatic and manual removal of duplicates. For the exclusion of studies, two new libraries were created in EndNote (primary inclusion/primary exclusion), and the studies selected by each researcher in the primary selection stage were combined. Then, original articles were identified and secondary study selection was performed. After duplicate removal, the titles and abstracts of the remaining articles were reviewed for primary selection. The full texts of the articles selected in the primary selection process were obtained and read, following which the final selection procedure was completed by considering the inclusion and exclusion criteria of the present study. This entire process was performed independently by the two researchers. The final studies were selected after the two researchers crosschecked their findings and reached an agreement on the selection.

\section{Data synthesis}

To investigate whether topical fluoride gel application is effective for the prevention of dental caries in the

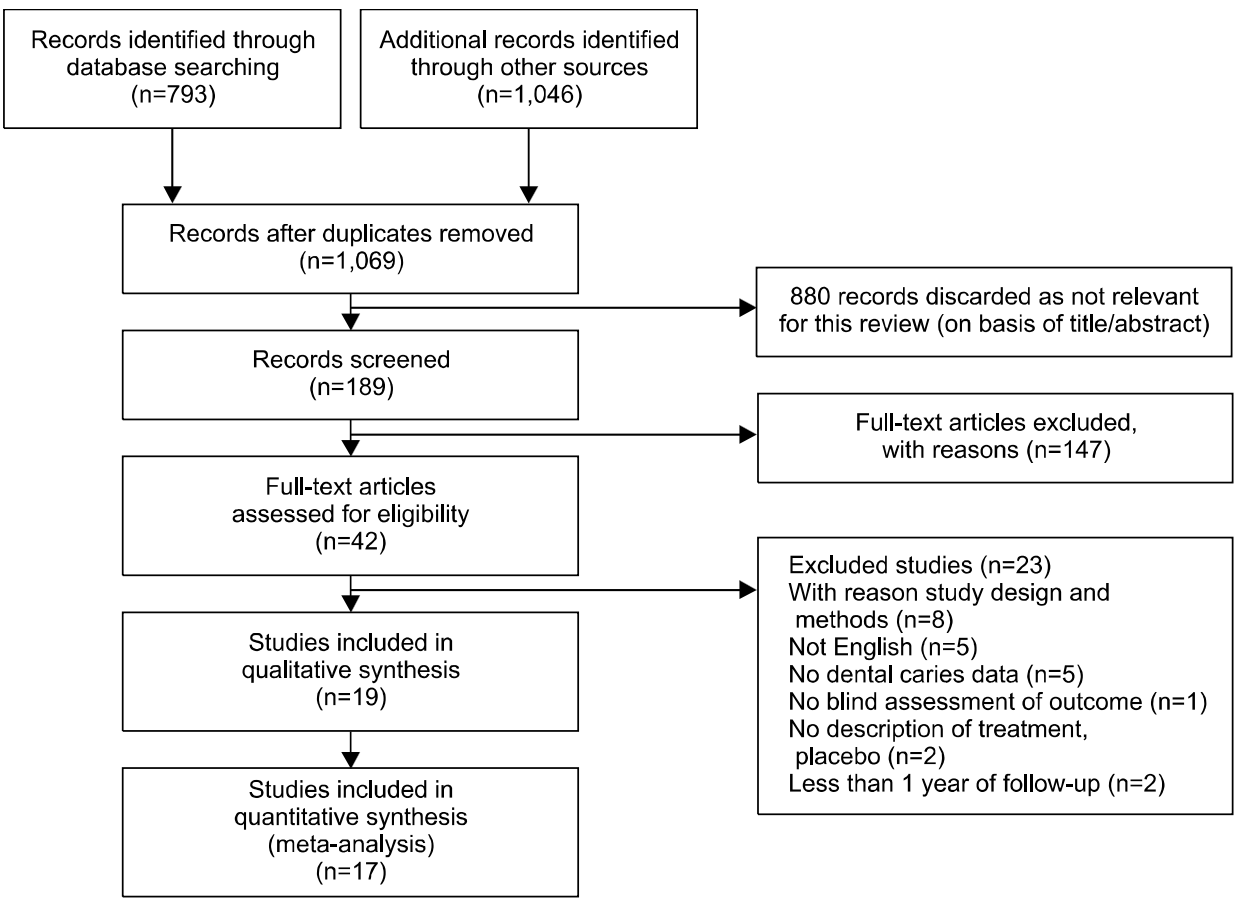

Fig. 1. Study flow diagram from 2018 search 


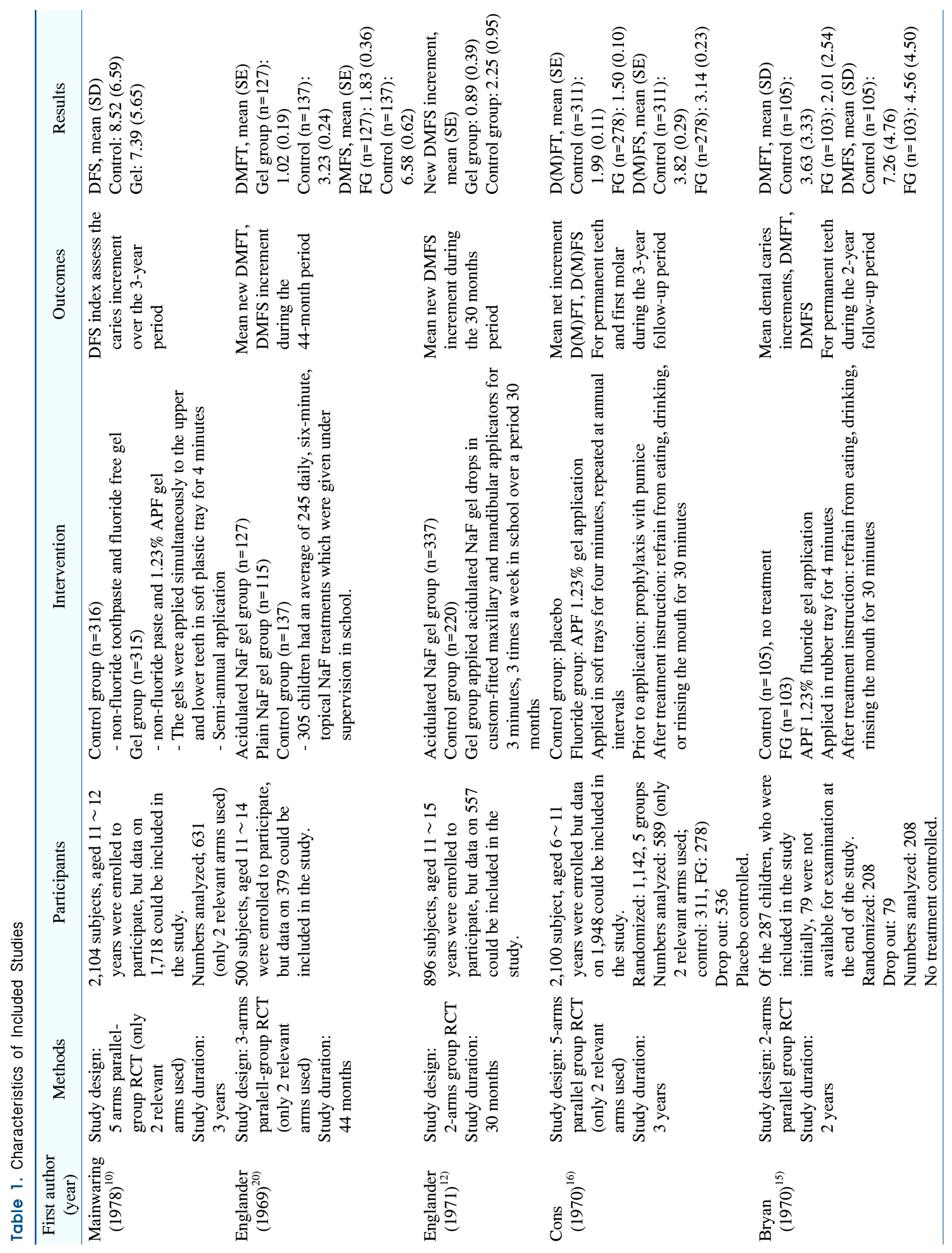




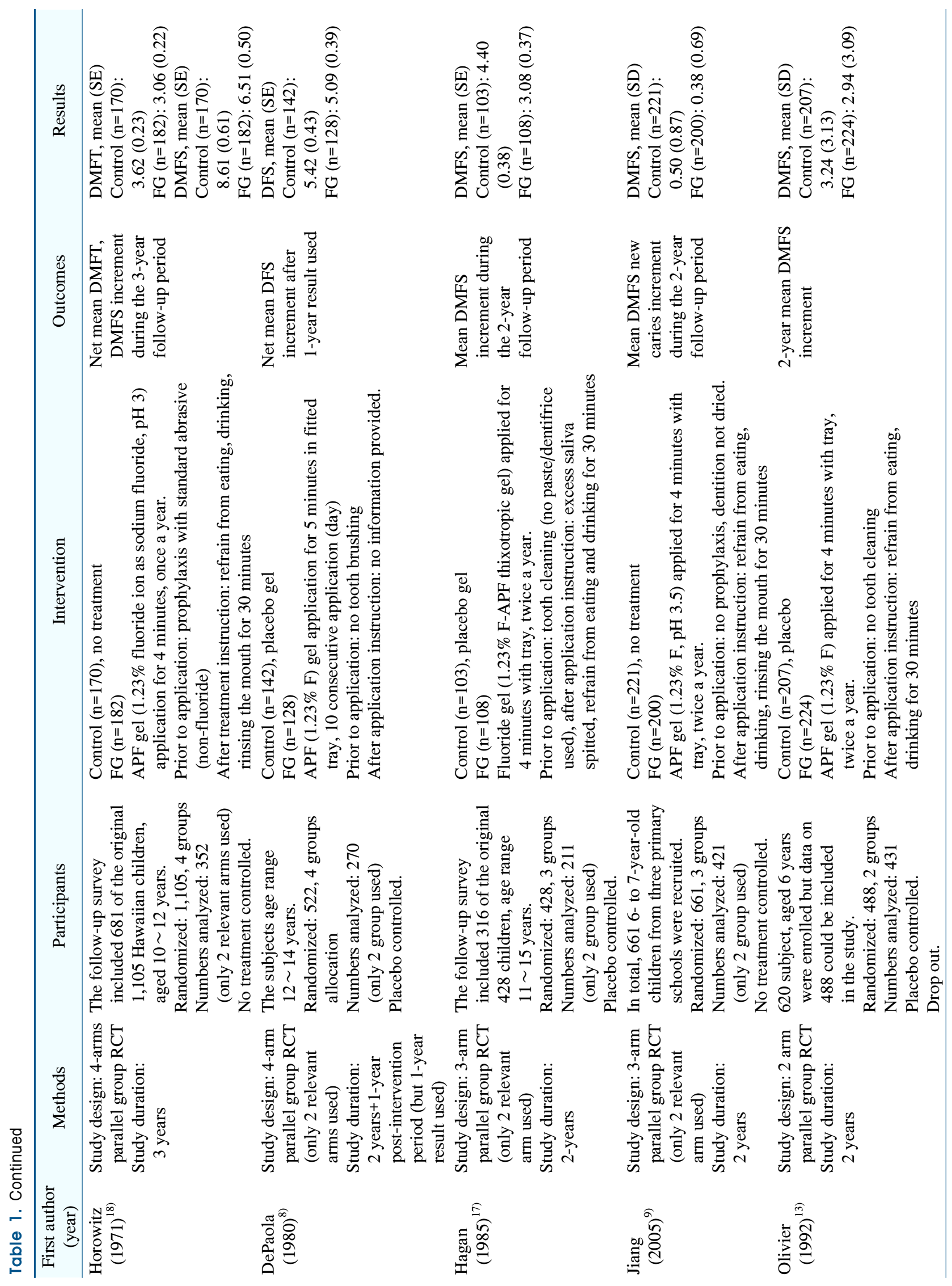




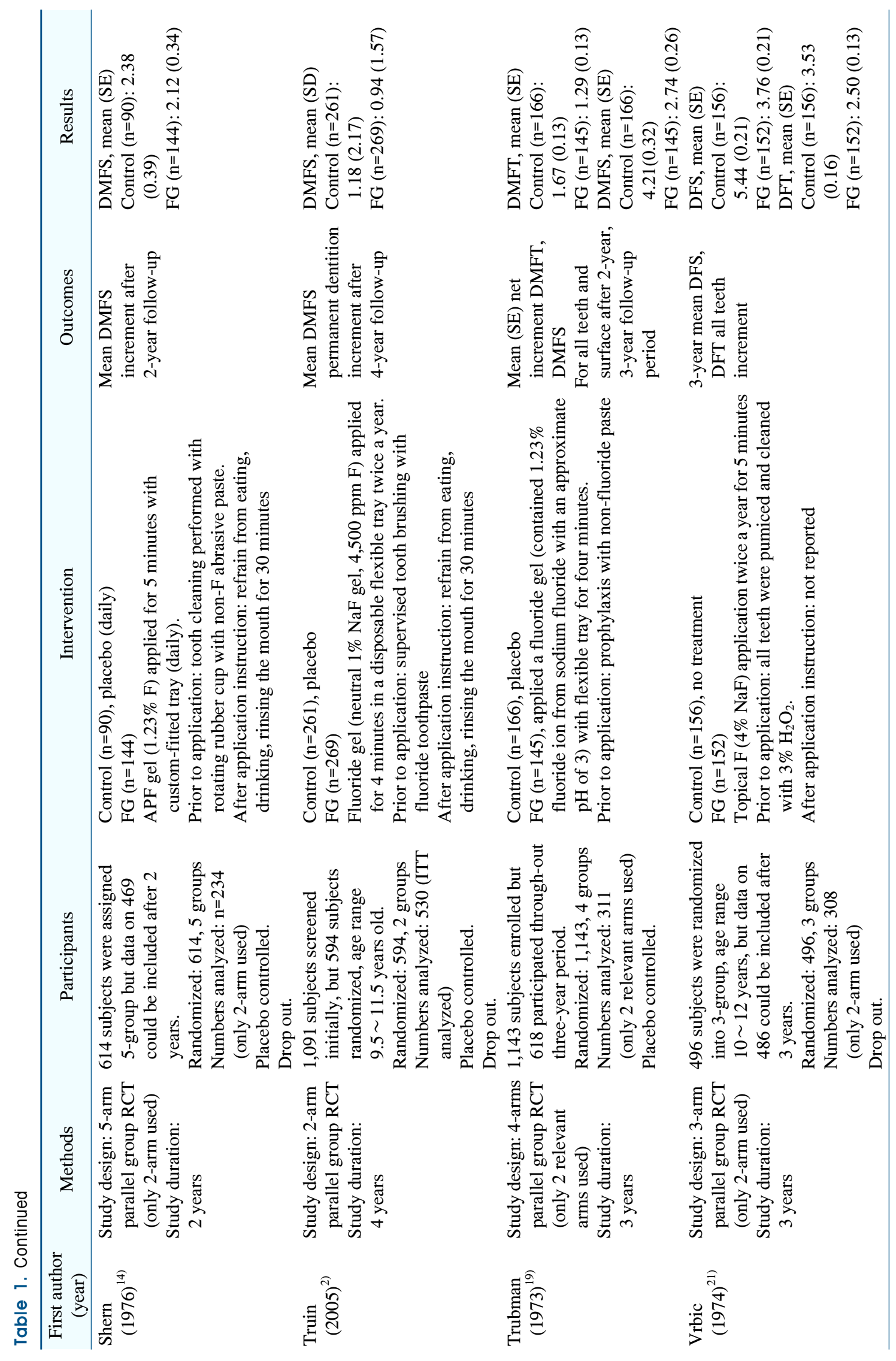




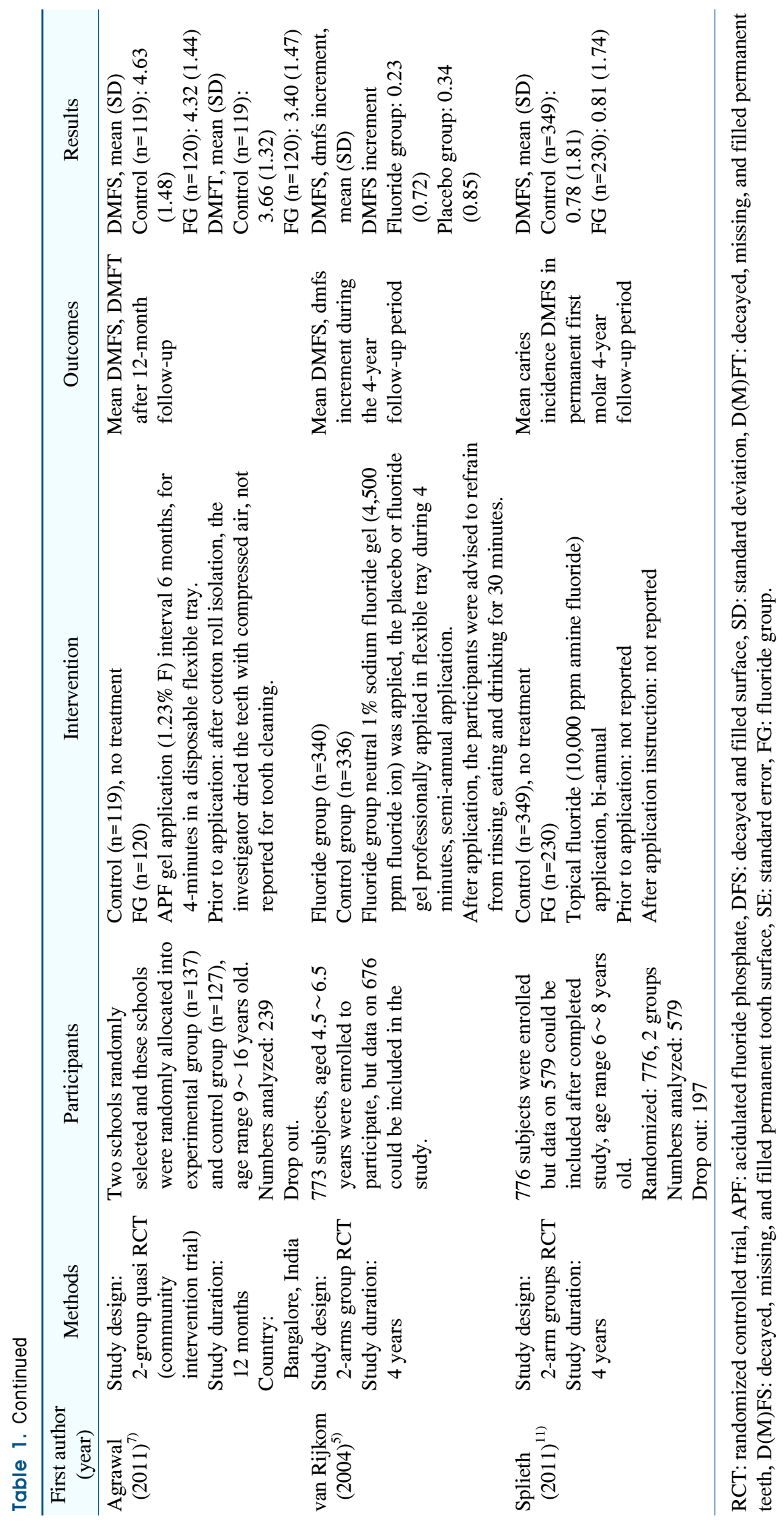


permanent teeth of children and adolescents, we performed a meta-analysis for quantitative synthesis of the data from the final 17 studies. Q statistics were used to determine the degree of heterogeneity of the selected studies, and meta-analysis using a random effects model was performed depending on the results of heterogeneity testing. Then, a forest plot was generated. All analyses were performed using Review Manager (RevMan) statistical analysis software (ver. 5.3; The Nordic Cochrane Centre, The Cochrane Collaboration, Copenhagen, Denmark).

\section{Results}

\section{Search results}

Using the search strategy, 793, 286, and 760 studies were identified from the PubMed, EMBASE, and Cochrane Library databases, respectively. Among the 1,839 studies, 770 duplicate studies were excluded, leaving 1,069 studies. Subsequently, 880 studies were excluded after a review of the titles and abstracts. From the remaining 189 studies, 42 original articles were identified. After reading the full text of these original articles, the inclusion and exclusion criteria were carefully applied. Consequently, 17 studies were selected for quantitative synthesis after excluding 25 studies for the following reasons: inappropriate design and/or study methods $(n=8)$, inability to extract the outcome indices $(n=5)$, lack of blinding during measurement of the outcome indices $(n=1)$, no description about the control group intervention $(n=2)$, follow-up period of less than 1 year $(n=2)$, and publication in languages other than English $(n=5)$. Fig. 1 shows the flow diagram (PRISMA) for the study selection process.

\section{Meta-analysis for estimation of results}

For qualitative review of the selected studies, the source, methods, subjects, intervention methods, outcome indices, and results were summarized and are presented in the evidence table in Table $1^{2,5,7-21)}$.

The finding of meta-analysis revealed that 16 studies found differences in the $\mathrm{D}(\mathrm{M}) \mathrm{FS}$ and $\mathrm{D}(\mathrm{M}) \mathrm{FT}$ indices between experimental (fluoride gel application) and control groups. Specifically, the indices were lower in the experimental group than in the control group. In particular, a study by Englander et al. ${ }^{20)}$ showed the largest mean

\begin{tabular}{|c|c|c|c|c|c|c|c|c|c|c|c|c|}
\hline \multirow{2}{*}{$\begin{array}{l}\text { Study or subgroup } \\
\text { Agrawal (2011) }\end{array}$} & \multicolumn{3}{|c|}{ Experimental } & Mean & Control & Total & \multicolumn{2}{|c|}{$\begin{array}{c}\text { Mean difference } \\
\text { Weight (\%) IV, random, } 95 \% \mathrm{Cl}\end{array}$} & \multicolumn{4}{|c|}{$\begin{array}{c}\text { Mean difference } \\
\text { IV, random, } 95 \% \mathrm{Cl}\end{array}$} \\
\hline & 4.32 & 1.44 & 120 & 4.63 & 1.49 & 119 & 8.1 & $-0.31[-0.68,0.06]$ & & & & \\
\hline Bryan (1970) & 4.56 & 4.5 & 103 & 7.26 & 4.76 & 105 & 3.2 & $-2.70[-3.96,-1.44]$ & & & & \\
\hline Cons (1970) & 3.14 & 3.83 & 278 & 3.82 & 5.11 & 311 & 5.8 & $-0.68[-1.40,0.04]$ & & & & \\
\hline DePaola (1980) & 5.09 & 4.41 & 128 & 5.42 & 5.12 & 142 & 3.7 & $-0.33[-1.47,0.81]$ & & & & \\
\hline Englander (1969) & 1.83 & 4.06 & 127 & 6.58 & 7.26 & 137 & 2.8 & $-4.75[-6.16,-3.34]$ & & - & & \\
\hline Englander (1971) & 1.57 & 2.94 & 337 & 2.2 & 2.97 & 220 & 7.2 & $-0.63[-1.13,-0.13]$ & & & 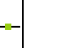 & \\
\hline Hagan (1985) & 3.08 & 3.85 & 108 & 4.4 & 3.86 & 103 & 4.1 & $-1.32[-2.36,-0.28]$ & & & & \\
\hline Horowitz (1971) & 6.51 & 6.75 & 182 & 8.61 & 7.95 & 170 & 2.4 & $-2.10[-3.65,-0.55]$ & & - & & \\
\hline Jiang (2005) & 0.38 & 0.69 & 200 & 0.5 & 0.87 & 221 & 9.2 & $-0.12[-0.27,0.03]$ & & & & \\
\hline Mainwaring (1978) & 7.39 & 5.65 & 315 & 8.52 & 6.59 & 316 & 4.5 & $-1.13[-2.09,-0.17]$ & & & & \\
\hline Olivier (1992) & 2.94 & 3.09 & 224 & 3.24 & 3.13 & 207 & 6.7 & $-0.30[-0.89,0.29]$ & & & & \\
\hline Shern (1976) & 2.12 & 4.08 & 144 & 2.38 & 3.7 & 90 & 4.2 & $-0.26[-1.27,0.75]$ & & & & \\
\hline Splieth (2011) & 0.81 & 1.74 & 230 & 0.78 & 1.81 & 349 & 8.6 & $0.03[-0.26,0.32]$ & & & & \\
\hline Trubman (1973) & 2.74 & 3.13 & 145 & 4.21 & 4.12 & 166 & 5.3 & $-1.47[-2.28,-0.66]$ & & - & & \\
\hline Truin (2005) & 0.94 & 1.57 & 269 & 1.18 & 2.17 & 261 & 8.4 & $-0.24[-0.56,0.08]$ & & & & \\
\hline van Rijkom (2004) & 0.23 & 0.72 & 372 & 0.34 & 0.85 & 360 & 9.3 & $-0.11[-0.22,0.00]$ & & & & \\
\hline Vrbic (1974) & 3.76 & 2.59 & 152 & 5.44 & 2.62 & 156 & 6.7 & $-1.68[-2.26,-1.10]$ & & - & & \\
\hline Total $(95 \% \mathrm{Cl})$ & & & 3,434 & & & 3,433 & 100.0 & $-0.76[-1.03,-0.48]$ & & & 1 & \\
\hline \multicolumn{9}{|c|}{ Heterogeneity: $\operatorname{Tau}^{2}=0.21 ;$ chi $^{2}=114.12, d f=16(p<0.00001) ; I^{2}=86 \%$} & -10 & -5 & 0 & 5 \\
\hline \multicolumn{9}{|c|}{ Test for overall effect: $Z=5.32(p<0.00001)$} & & $\begin{array}{l}\text { Favours } \\
\text { perimental] }\end{array}$ & & $\begin{array}{l}\text { Favours } \\
\text { [control] }\end{array}$ \\
\hline
\end{tabular}

Fig. 2. Comparison fluoride gel versus control (placebo or no treatment), outcome D(M)FS increment nearest to $1 \sim 4$ years (17 trials). SD: standard deviation, Cl: confidence interval. 
difference $(\mathrm{MD})$ of -4.75 (95\% confidence interval [CI]: -6.16 to -3.34 ) in the $\mathrm{D}(\mathrm{M}) \mathrm{FS}$ index (random effects model). A study by Bryan and Williams ${ }^{15)}$ also showed an MD of -2.70 (95\% CI: -3.96 to -1.44$)$ in the D(M)FS index. On the other hand, a study by Splieth et al. ${ }^{11)}$ found almost no difference between the two groups (MD: 0.03, $95 \%$ CI: -0.26 to 0.32 ).

Quantitative synthesis (meta-analysis) of the 17 studies involved the analysis of data from 3,434 subjects in experimental groups and 3,433 subjects in control groups. The overall MD was -0.76 (95\% CI: -1.03 to -0.48$)$, which indicated that the $\mathrm{D}(\mathrm{M}) \mathrm{FS}$ index was lower in the experimental group than in the control group, and that topical fluoride gel application was effective in preventing dental caries development in the permanent teeth of children and adolescents ( $\mathrm{p}<0.001$ ) (Fig. 2) ${ }^{2,5,7-21)}$. Moreover, a meta-analysis of $\mathrm{D}(\mathrm{M}) \mathrm{FT}$ data from a total of seven studies involved the analysis of data from 1,117 subjects in experimental groups and 1,172 subjects in control groups. The overall MD was -0.88 (95\% CI: -1.32 to -0.44, p <0.001) (Fig. 3) 7,15,16,18-21) . The two analyses showed an $\mathrm{I}^{2}$ value of $86 \%$. According to the classification proposed by the Cochrane Handbook for Systematic Reviews ${ }^{22)}$, the interpretation of $\mathrm{I}^{2}$ values is as follows: $0 \sim$ $40 \%$, not important; $30 \sim 60 \%$, moderate heterogeneity; $50 \sim 90 \%$, substantial heterogeneity; and 75 100\%, considerable heterogeneity.

We also performed a subgroup analysis to determine whether the effects of fluoride application differed if patients received oral prophylaxis (self or professional) before fluoride gel application. The overall MD for the experimental and control groups in studies where prophylaxis was performed before fluoride gel application was -1.03 (95\% CI: -1.55 to -0.51$)$, whereas that for the experimental and control groups in studies where no oral prophylaxis was performed before fluoride gel application was -1.37 (95\% CI: -2.18 to -0.56 ). Therefore, the two groups showed slightly larger differences when studies without oral prophylaxis before fluoride gel application were considered; however, the difference was not statistically significant (Fig. 4) $2,5,7-21)$.

\section{Risk of bias in the included studies}

We used the Cochrane Collaboration's tool for assessing the risk of bias (Cochrane Handbook for Systematic Review of Intervention, ver. 5.1) ${ }^{22)}$ in seven domains, namely sequence generation, allocation concealment, blinding of participants/personnel, blinding during outcome assessment, incomplete outcome data, selective reporting, and other bias. We described information reported in the study and assigned a judgment relating to the risk of bias for that entry. Then, we assigned the studies into the following categories: low risk of bias (plausible bias unlikely to seriously alter the results, low risk of bias assessed for all seven domains); high risk of bias (plausible bias that seriously weakens the results, high risk of bias assessed for at least one domain); and unclear risk of bias (plausible bias that raises some doubt about the results,

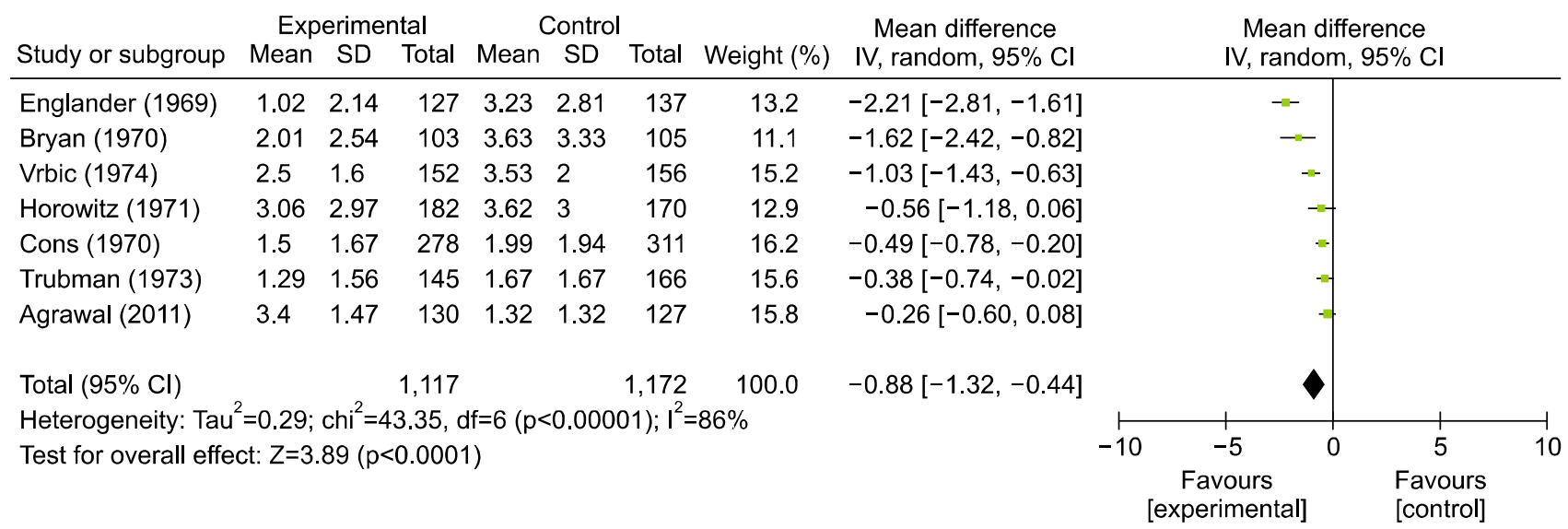

Fig. 3. Comparison fluoride gel versus control (placebo or no treatment), outcome D(M)FT increment nearest to $1 \sim 4$ years (7 trials). SD: standard deviation, $\mathrm{Cl}$ : confidence interval. 


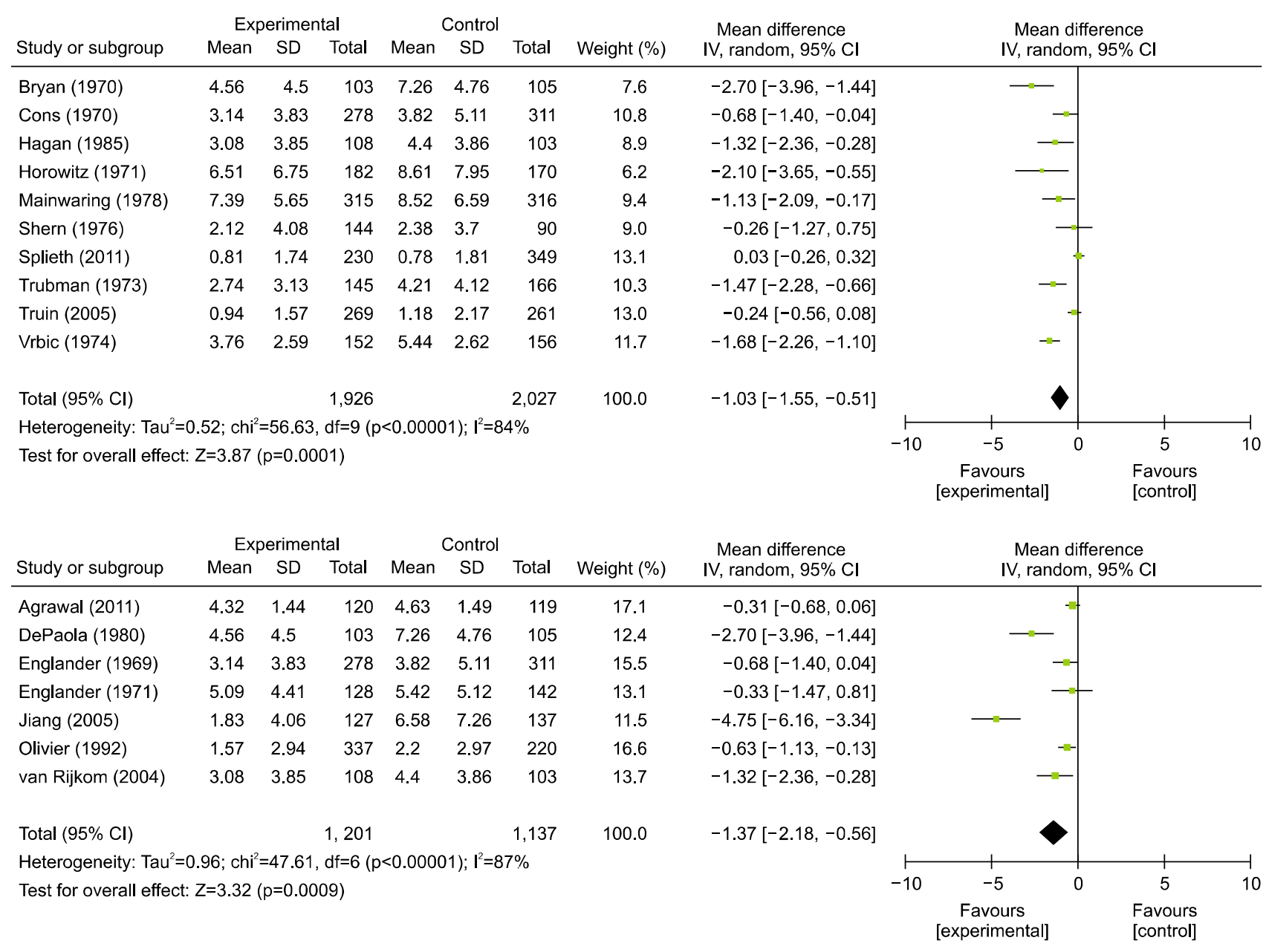

Fig. 4. Comparison prophylaxis vs no prophylaxis ,outcome D(M)FS index difference before fluoride gel application. SD: standard deviation, $\mathrm{Cl}$ : confidence interval.

unclear risk of bias assessed for at least one domain, with no judgment of high risk for any domain). The results of bias risk assessments are shown in Fig. $5^{2,5,7-21)}$.

\section{Discussion}

Over the past few decades, the prevalence of dental caries has gradually decreased, but a significant number of children and adolescents are still afflicted with this disease. Thus, if not treated early, it can cause severe pain and negatively impact the oral health-related quality of life. Therefore, the prevention of dental caries, a chronic disease, is important, and additional preventive measures such as the use of sealants, regular topical fluoride application, use of auxiliary oral care products, and regular check-ups along with regular tooth-brushing after meals are recommended ${ }^{23-25)}$. Fluoride use was a part of dental caries prevention programs in the 1940s. The most initial measure was public water fluoridation in the USA and Canada, which was subsequently adapted by other countries worldwide $^{26)}$. Currently, fluoride is widely used as a prophylactic agent for early dental caries, and it is extensively used in clinical settings as a form of preventive therapy on the basis of reports of its ability to prevent initial carious lesions via plaque removal, removal of demineralized enamel without exposing the tooth surface to cariogenic challenge, and promotion of enamel remineralization by minimizing mineral loss ${ }^{3,6,27)}$. A low concentration of fluoride must remain on the enamel surface in the oral cavity, and good effects can be achieved if plaque, saliva, and oral soft tissues act as reservoirs and remain loosely bound to the enamel surface. Moreover, 


\begin{tabular}{|c|c|c|c|c|c|c|}
\hline Study or subgroup & A & B & \begin{tabular}{l|l}
$C$ & $D$
\end{tabular} & $D \mid E$ & $F$ & $G$ \\
\hline Agrawal (2011) & (?) & ๑ & $\odot \mathrm{e}$ & כ) & $\oplus$ & (3) \\
\hline Bryan (1970) & $\odot$ & (2) & $\odot ?$ & (3) (?) & $\oplus$ & (?) \\
\hline Cons (1970) & (?) & (2) & (?) $€$ & $\oplus 0$ & $\oplus$ & (2) \\
\hline DePaola (1980) & (?) & (?) & $\oplus \oplus$ & $\oplus \Theta$ & $\oplus$ & (?) \\
\hline Englander (1969) & (2) & (?) & $\odot ?$ & ?) & (?) & (?) \\
\hline Englander (1971) & (?) & (?) & $\odot ?$ & ?) & $\oplus$ & (?) \\
\hline Hagan (1985) & (?) & (?) & $\oplus$ & (†) (?) & $\oplus$ & (?) \\
\hline Horowitz (1971) & $\Theta$ & $\odot$ & $\odot ?$ & ?) & (?) & $\Theta$ \\
\hline Jiang (2005) & (2) & (2) & ○? & ?) & (?) & (2) \\
\hline Mainwaring (1978) & (?) & (?) & $\oplus$ & (1) (?) & $\oplus$ & (?) \\
\hline Olivier (1992) & (?) & (?) & (?) $€$ & $\oplus$ † & $\oplus$ & (?) \\
\hline Shern (1976) & (2) & (?) & $\oplus \oplus$ & (7) (?) & $\oplus$ & (?) \\
\hline Splieth (2011) & (?) & $\odot$ & $\oplus \oplus$ & $\oplus \oplus$ & $\oplus$ & (?) \\
\hline Trubman (1973) & (?) & (?) & $\oplus \oplus$ & $\oplus$ & $\oplus$ & (?) \\
\hline Truin (2005) & $\odot$ & $\oplus$ & $\oplus \Subset$ & †) & (?) & $\odot$ \\
\hline van Rijkom (2004) & $\oplus$ & ๑) & $\oplus$ & $\oplus \oplus$ & (?) & $\oplus$ \\
\hline Vrbic (1974) & (?) & (?) & (?) & $\oplus \mid \oplus$ & $\oplus$ & $\Theta$ \\
\hline \multicolumn{7}{|l|}{ Risk of bias legend } \\
\hline $\begin{array}{l}\text { (A) Random sequer } \\
\text { (B) Allocation conce } \\
\text { (C) Blinding of parti } \\
\text { (D) Blinding of outc } \\
\text { (E) Incomplete outc } \\
\text { (F) Selective reporti } \\
\text { (G) Other bias }\end{array}$ & & & ction & $\begin{array}{l}\text { n } \\
\text { s) }\end{array}$ & & $\begin{array}{l}\text { ias) } \\
\text { formance bias) } \\
\text { n bias) }\end{array}$ \\
\hline
\end{tabular}

Fig. 5. 'Risk of bias' summary: review authors' judgements about each risk of bias item for each included study.

continued exposure to low concentrations of fluoride through the consumption of fluoride-containing water, salt, and milk is also an excellent method, while the application of highly concentrated fluoride gels and varnishes by clinical professionals has also been reported as a noteworthy method ${ }^{28)}$. However, some studies reported that tooth-brushing at least twice a day with toothpaste containing fluoride was more important ${ }^{24,29)}$, whereas one study reported that there were no significant differences in the caries prevention rate between a group that received fluoride treatment and a group that did not ${ }^{11)}$. Therefore, there are inconsistencies among study results, and it is possible that the method of fluoride use would be different in actual clinical settings, which would also result in differences in effects. Moreover, effects may differ under the influence of the following factors: professional cleaning or self-cleaning of teeth before topical fluoride application, mode of application (self or professional), pretreatment procedures (air drying of the tooth surface, saliva control), patient compliance with treatment, and compliance with post-treatment instructions.
To derive latest evidence on the preventive effects of topical fluoride gel application on dental caries in the permanent teeth of children and adolescents, we performed a meta-analysis by systematically reviewing RCTs. Moreover, we performed a subgroup analysis to investigate whether the effects of topical fluoride gel application differed if teeth were professionally cleaned or self-cleaned before application. We found that the $\mathrm{D}(\mathrm{M}) \mathrm{FS}$ and $\mathrm{D}(\mathrm{M}) \mathrm{FT}$ indices were lower in the experimental group than in the control group (placebo or no treatment). In other words, topical fluoride application on permanent teeth was effective in preventing dental caries. In a recent systematic review conducted by Marinho et al. ${ }^{1)}$, a total of 25 studies were quantitatively synthesized (total of 8,479 subjects). The pooled estimate for the $\mathrm{D}(\mathrm{M}) \mathrm{FS}$ preventive fraction was $0.28 \%$ (95\% CI: 0.19 to $0.36, \mathrm{p}<0.001$ ), indicating a large preventive effect. Therefore, the effects of topical fluoride gel application were similar to those found in the present review. However, in the previous meta-analysis, studies conducted by Truin and van't Hof $^{2)}$ and Jiang et al. ${ }^{9)}$ in 2005 were the latest studies. The present study included two articles from $2011^{7,11)}$, and a total of 17 RCTs were finally included after the application of stringent inclusion and exclusion criteria. Thirteen of these 17 studies were also included in the previous review of $2015^{2,5,8-10,12-19)}$, while four were newly added ${ }^{7,11,20,21)}$. Also, in the previous study, the inclusion criteria did not specifically mention the fluoride application method. For example, it included studies that used various methods such as fluoride application on a toothbrush by a professional, the use of foam-type fluoride, fluoride application simultaneous with professional tooth cleaning with fluoride-containing toothpaste, and combined programs including oral health education ${ }^{1,30-32)}$. However, we applied stringent inclusion and exclusion criteria for investigating the effects of topical fluoride gels, which are extensively used in clinical settings. Moreover, we only considered permanent teeth and excluded studies that assessed primary teeth ${ }^{33,34)}$.

In the present review, we also assessed the effects of topical fluoride application after professional prophylaxis or self-cleaning of teeth. There was no significant difference in the effects of fluoride gel application between 
studies where oral prophylaxis was performed before fluoride gel application and studies where no oral prophylaxis was performed before fluoride gel application. It remains unclear whether the biofilm should be completely eliminated before the application of fluoride, and whether fluorides should be applied in noncompliant children. However, according to the fluoride application guidelines published by the American Academy of Pediatric Dentistry, it was reported that prophylaxis prior to fluoride application did not increase efficacy compared with no prophylaxis. It is commonly accepted that regular tooth-brushing twice a day is adequate ${ }^{35)}$. Therefore, if patients are sufficiently not compliant, there should not be any problem without oral prophylaxis. Furthermore it would be necessary to use the findings of the present study to actively raise awareness about the caries-prevention effects of topical fluoride application among patients and guardians.

This study has some limitations. First, publication bias could not be assessed for the selected studies because unpublished gray literature was not included. Second, only four recent RCTs that were not included in the previous systematic review were included in the present one. Third, there was large heterogeneity among studies, but accurate heterogeneity assessments were not performed. Reviews including more of the latest studies are necessary, with additional analyses to enhance the present evidence, such as accurate heterogeneity assessments and sensitivity analyses. In a 2016 systematic review by Gao et al. ${ }^{36}$, differences in effects according to the type of fluoride were studied, and 5\% sodium fluoride varnish was reported to have the best effect on the remineralization of early enamel caries, while $38 \%$ silver diamine fluoride solution was effective in arresting dentin caries ${ }^{36)}$. However, relevant RCTs on this topic are very limited. Future studies should consider the type of fluoride, number of topical fluoride applications, duration of topical application, concentration of fluoride, differences in effects with different methods, and adverse effects associated with fluoride use.

The biggest strength of our study is the assessment of only topical fluoride gel application, which is used most extensively in the clinical setting, for presenting evidence to prove its effectiveness. Moreover, we also conducted a subgroup analysis to assess differences in caries-prevention effects when topical fluorides were applied after professional cleaning or self-cleaning of teeth. The results of our subgroup analysis showed that the effects of fluoride gel application did not differ when patients received oral prophylaxis before fluoride application. This reaffirmed the results of previous studies, which showed that prophylaxis did not enhance the effects of fluoride application. On the basis of our findings, fluoride application should be recommended as a preventive treatment for tooth decay in children and adolescents. The effects of topical fluoride application on other conditions such as hyperesthesia, cervical abrasion in adults or the elderly, and dental root caries should be determined in future systematic reviews designed to provide highly reliable evidence.

\section{Notes}

\section{Conflict of interest}

No potential conflict of interest relevant to this article was reported.

\section{Ethical approval}

Not applicable.

\section{ORCID}

Kyung-Yi Do, https://orcid.org/0000-0002-7690-6314

Eun-Sun Lee, https://orcid.org/0000-0002-3431-3932

\section{References}

1. Marinho VC, Worthington HV, Walsh T, Chong LY: Fluoride gels for preventing dental caries in children and adolescents. Cochrane Database Syst Rev (6): CD002280, 2015. https://doi.org/10.1002/14651858.CD002280

2. Truin GJ, van't Hof MA: Professionally applied fluoride gel in low-caries 10.5-year-olds. J Dent Res 84: 418-421, 2005. https://doi.org/10.1177/154405910508400504

3. ten Cate JM: Current concepts on the theories of the mechanism of action of fluoride. Acta Odontol Scand 57: 325-329, 1999. 
4. Jung EY, Kim MJ, Kim YJ, et al.: Associated factors of the approval for the community water fluoridation program. J Dent Hyg Sci 13: 29-35, 2013.

5. van Rijkom HM, Truin GJ, van 't Hof MA: Caries-inhibiting effect of professional fluoride gel application in low-caries children initially aged 4.5-6.5 years. Caries Res 38: 115-123, 2004. https://doi.org/10.1159/000075935

6. Bonow ML, Azevedo MS, Goettems ML, Rodrigues CR: Efficacy of $1.23 \%$ APF gel applications on incipient carious lesions: a double-blind randomized clinical trial. Braz Oral Res 27: 279-285, 2013. https://doi.org/10.1590/S1806-83242013000300007

7. Agrawal N, Pushpanjali K: Feasibility of including APF gel application in a school oral health promotion program as a caries-preventive agent: a community intervention trial. J Oral Sci 53: 185-191, 2011.

8. DePaola PF, Soparkar M, Van Leeuwen M, DeVelis R: The anticaries effect of single and combined topical fluoride systems in school children. Arch Oral Biol 25: 649-653, 1980.

9. Jiang H, Tai B, Du M, Peng B: Effect of professional application of APF foam on caries reduction in permanent first molars in 6-7-year-old children: 24-month clinical trial. J Dent 33: 469-473, 2005. https://doi.org/10.1016/j.jdent.2004.10.023

10. Mainwaring PJ, Naylor MN: A three-year clinical study to determine the separate and combined caries-inhibiting effects of sodium monofluorophosphate toothpaste and an acidulated phosphate-fluoride gel. Caries Res 12: 202-212, 1978. https://doi.org/10.1159/000260334

11. Splieth $\mathrm{CH}$, Berndt C, Alkilzy M, Treuner A: Efficacy of semiannual topical fluoride application in schoolchildren. Quintessence Int 42: 753-760, 2011.

12. Englander HR, Sherrill LT, Miller BG, Carlos JP, Mellberg JR, Senning RS: Incremental rates of dental caries after repeated topical sodium fluoride applications in children with lifelong consumption of fluoridated water. J Am Dent Assoc 82: 354-358, 1971.

13. Olivier M, Brodeur JM, Simard PL: Efficacy of APF treatments without prior toothcleaning targeted to high-risk children. Community Dent Oral Epidemiol 20: 38-42, 1992.

14. Shern RJ, Duany LF, Senning RS, Zinner DD: Clinical study of an amine fluoride gel and acidulated phosphate fluoride gel. Community Dent Oral Epidemiol 4: 133-136, 1976.

15. Bryan ET, Williams JE: The cariostatic effectiveness of a phosphate-fluoride gel administered annually to school children; final results. J Public Health Dent 30: 13-16, 1970.

16. Cons NC, Janerich DT, Senning RS: Albany topical fluoride study. J Am Dent Assoc 80: 777-781, 1970.

17. Hagan PP, Rozier RG, Bawden JW: The caries-preventive effects of full- and half-strength topical acidulated phosphate fluoride. Pediatr Dent 7: 185-191, 1985.

18. Horowitz HS, Doyle J: The effect on dental caries of topically applied acidulated phosphate-fluoride: results after three years. J Am Dent Assoc 82: 359-365, 1971.

19. Trubman A, Crellin JA: Effect on dental caries of self-application of acidulated phosphate fluoride paste and gel. J Am Dent Assoc 86: 153-157, 1973.

20. Englander HR, Carlos JP, Senning RS, Mellberg JR: Residual anticaries effect of repeated topical sodium fluoride applications by mouthpieces. J Am Dent Assoc 78: 783-787, 1969.

21. Vrbic V, Kosmelj B, Ravnik C: A 3-year study among Yugoslavian schoolchildren on caries reduction after topical application of $4 \%$ NaF-PO4. Community Dent Oral Epidemiol 2: 163-165, 1974.

22. Higgins JP, Altman DG, Gøtzsche PC, et al.: The Cochrane Collaboration's tool for assessing risk of bias in randomised trials. BMJ 343: d5928, 2011. https://doi.org/10.1136/bmj.d5928

23. Do KY, Lee ES, Lee KS: Association between excessive Internet use and oral health behaviors of Korean adolescents: a 2015 national survey. Community Dent Health 34: 183-189, 2017. https://doi.org/10.1922/CDH_4107Do07

24. Ferreira MA, Latorre Mdo R, Rodrigues CS, Lima KC: Effect of regular fluoride gel application on incipient carious lesions. Oral Health Prev Dent 3: 141-149, 2005.

25. Do KY: Impact of health risk factors on the oral health of Korean adolescents: Korea youth risk behavior web-based survey, 2013. J Dent Hyg Sci 16: 193-199, 2016. https://doi.org/10.17135/jdhs.2016.16.3.193

26. Miller FY, Campus G, Giuliana G, Piscopo MR, Pizzo G: Topical fluoride for preventing dental caries in children and adolescents. Curr Pharm Des 18: 5532-5541, 2012.

27. Jang SO, Choi EM, Oh SH, Kang MK, Kim KM: The surface characteristics of enamel according to fluoride application 
methods and frequency. J Dent Hyg Sci 11: 69-76, 2011.

28. O'Mullane DM, Baez RJ, Jones S, et al.: Fluoride and oral health. Community Dent Health 33: 69-99, 2016.

29. Stokes E, Ashcroft A, Burnside G, Mohindra T, Pine CM: Randomised controlled trial of the efficacy of a high-fluoride gel self-applied by toothbrushing in children at high caries risk. Caries Res 45: 475-485, 2011. https://doi.org/10.1159/000331205

30. Si Y, Guo Y, Yuan C, Xu T, Zheng SG: Comprehensive oral health care to reduce the incidence of severe early childhood caries (s-ECC) in urban China. Chin J Dent Res 19: 55-63, 2016. https://doi.org/10.3290/j.cjdr.a35698

31. Marthaler TM, König KG, Mühlemann HR: The effect of a fluoride gel used for supervised toothbrushing 15 or 30 times per year. Helv Odontol Acta 14: 67-77, 1970.

32. Treide A, Treide B: [Caries limiting effectiveness of newly developed fluoride-containing gels after 3 years clinical use in preschool years]. Stomatol DDR 38: 708-712, 1988.
German.

33. Jiang H, Bian Z, Tai BJ, Du MQ, Peng B: The effect of a bi-annual professional application of APF foam on dental caries increment in primary teeth: 24-month clinical trial. J Dent Res 84: 265-268, 2005. https://doi.org/10.1177/154405910508400311

34. Andruskeviciene V, Milciuviene S, Bendoraitiene E, et al.: Oral health status and effectiveness of caries prevention programme in kindergartens in Kaunas city (Lithuania). Oral Health Prev Dent 6: 343-348, 2008.

35. Maguire A: ADA clinical recommendations on topical fluoride for caries prevention. Evid Based Dent 15: 38-39, 2014. https://doi.org/10.1038/sj.ebd.6401019

36. Gao SS, Zhang S, Mei ML, Lo EC, Chu CH: Caries remineralisation and arresting effect in children by professionally applied fluoride treatment: a systematic review. BMC Oral Health 16: 12, 2016.

https://doi.org/10.1186/s12903-016-0171-6 